\title{
The pathogenesis of respiratory viral infection
}

\author{
R. B. HeATH \\ M.D., F.R.C.Path.
}

The Joint Department of Virology of the Medical Colleges of St Bartholomew's and The London Hospitals

\begin{abstract}
Summary
Sendai virus is used as a model to study the histological response and the mechanism of recovery of mice from infection. Although mice infected intranasally do not appear ill, the ciliated columnar cells of the bronchial epithelium undergoes rapid necrosis followed later by repair. Interferon is produced at an early stage of infection and antibody formation follows later. Cyclophosphamide given to mice infected with Sendai virus abolishes the humoral antibody response but not interferon production; the mice develop pneumonic lesions. They cannot eliminate virus and they die. On the other hand, deprivation of ' $T$ ' lymphocytes by thymectomy, irradiation and bone marrow reconstitution renders mice more susceptible to Sendai virus but they do not die. The humoral response is thus apparently helped by ' $T$ ' lymphocytes and interferon production is not vital for recovery.
\end{abstract}

\section{Introduction}

Sendai virus is a common murine respiratory pathogen. It is endemic in many colonies of laboratory mice including those of certain highly reputable commercial breeders. It is an interesting virus, because if it is inoculated intranasally into susceptible mice there is no overt manifestation of clinical illness. However, histological and virological examination of inoculated animals reveals that they have experienced an upper respiratory tract infection from which they recover normally. Sendai virus infection of mice is thus an excellent model of acute respiratory viral infection of man. It is quite different from the adapted influenza infection of mice which induces viral pneumonic illness: a pathology which mercifully is rarely seen in man.

In this paper, some of the studies of Sendai virus infection of mice which have led to and are leading to a better understanding of the pathogenesis of acute respiratory viral infection will be briefly reviewed.

\section{The pathology of the infection}

As mentioned above, inoculation of mice with Sendai virus does not produce overt evidence of disease. However, the fact that these inoculated mice are ill can be deduced from the finding that they gain weight at a significantly slower rate than do mock inoculated control mice. Histological examination of lungs removed at intervals after inoculation reveals the characteristic features of acute respiratory viral infection (Robinson, Cureton and Heath, 1968). Within $24 \mathrm{hr}$ of inoculation, early damage to patches of the airway mucosa is apparent. Immunofluorescence staining for viral antigens shows that this early damage is associated with viral replication which is confined to the mucosa; there is no involvement of alveolar cells. By the third day of the infection the involved areas of the mucosa are completely destroyed and this is associated with intense inflammatory changes in the underlying mucosa (Fig. 1b). The damaging effect of the virus declines towards the end of the first week of the infection. By the beginning of the second week if is apparent that the infection is over, for at that time repair of damaged areas of mucosa can be seen. The submucosa underlying these areas is now congested with mononuclear cells of the lymphoid series (Fig. 1c). All of the damaged mucosa is completely repaired by the end of the second week (Fig. 1d). The pathological changes of the airways that are seen in the model system are essentially similar to those caused by influenza in mice (Andrewes, Laidlaw and Smith, 1934), ferrets (Francis and Stuart-Harris, 1938) and man (Hers, Masurel and Mulder, 1958).

The remarkable feature of these changes is not the infectious process itself but the fact that it is so abruptly stopped soon after initiation and, in particular, is prevented from extending to involve the whole of the susceptible mucosa. This clearly indicates that respiratory viral infection induces powerful local defence responses, which rapidly bring about its termination. Investigations related to evaluation of these defences will now be briefly described.

\section{Defence against the primary infection}

Virus growth and both interferon and antibody production occurring during the course of the infection can be determined by assay of serum and suspensions of lungs taken at intervals after inoculation (Robinson et al., 1968). The data obtained 


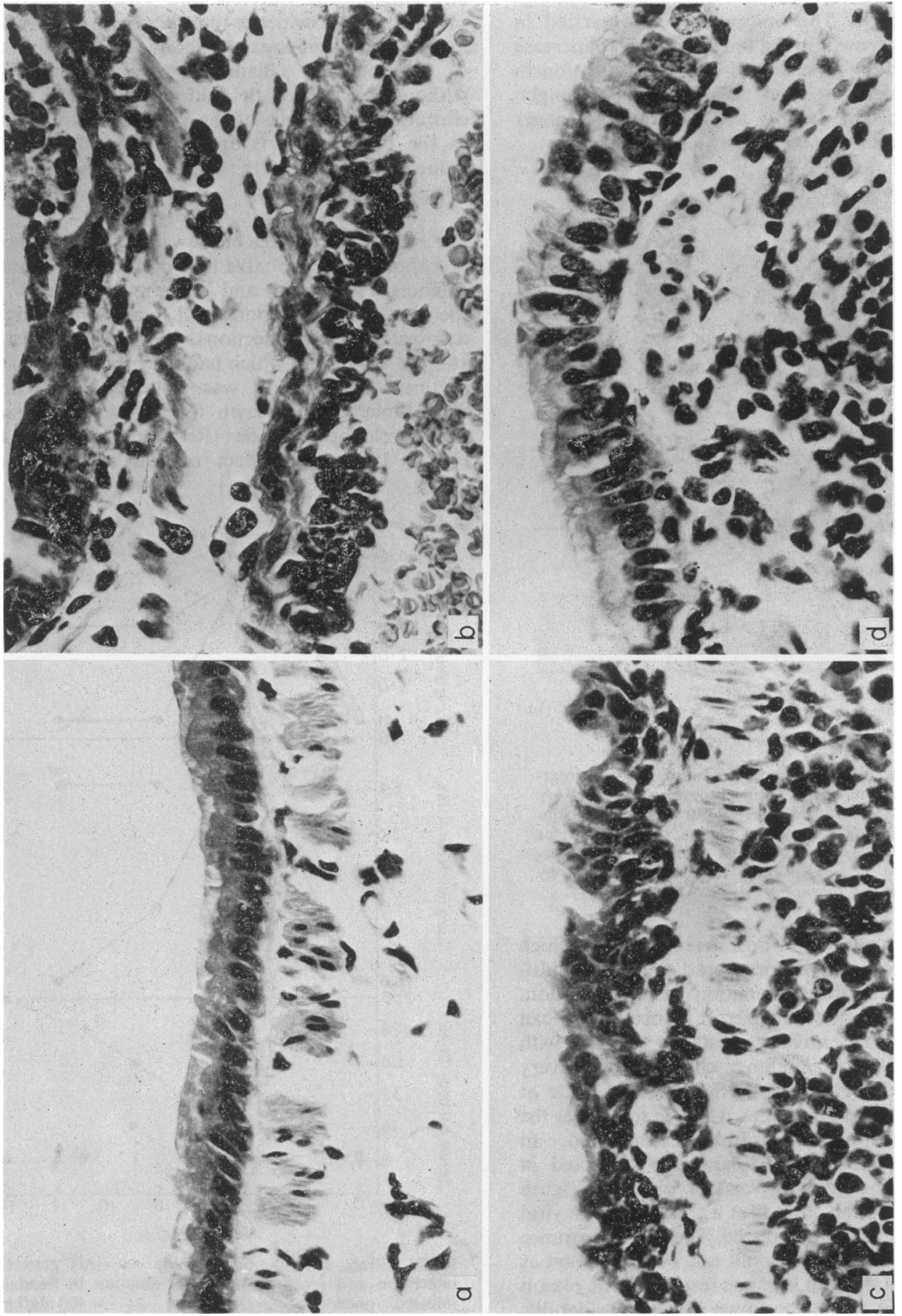

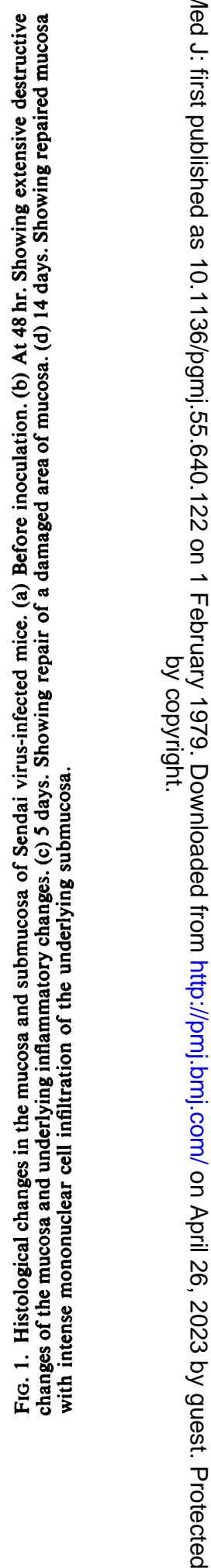


can be correlated with the histological findings described above. The findings are summarized in Fig. 2. Virus growth in the lungs is found to increase rapidly, reaching a peak level on the third to fourth day of the infection. Then, perhaps not surprisingly, virus titres decline rapidly and virus can no longer be detected after the eighth day.

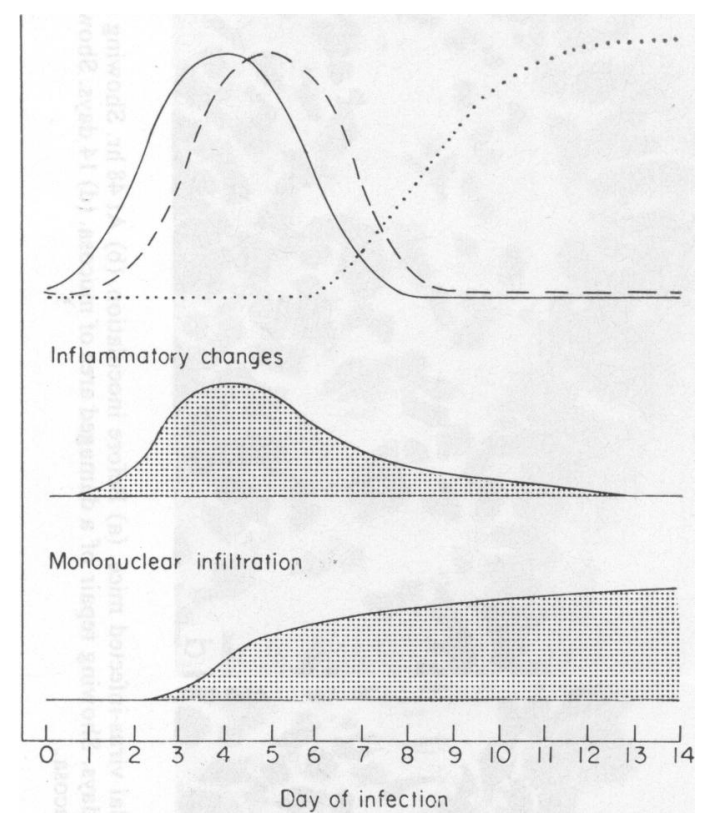

FIG. 2. Diagrammatic summary of virus growth, interferon production, serum antibody levels, inflammatory changes and mononuclear cell infiltration of the submucosa occurring during the course of Sendai virus infection of mice. $-=$ Virus titres. - $--=$ Interferon titres. ...... = Antibody titres. (Reproduced by permission of the Journal of Medical Microbiology).

There are a number of host defence factors which can play some part in virus eradication that ultimately leads to the termination of the infection. Sendai virus is a good interferon inducer and it can be seen that this antiviral substance together with the non-specific inflammatory changes appear very early in the infection. Both those factors fade at approximately the same time, which is soon after the viral eradication process has commenced. In contrast, specific antibody cannot be detected in either serum or lung suspensions until the eighth day of the infection; that is at a time when the viral eradication is nearly completed. The late appearance of detectable antibody should not be interpreted as indicative of a delayed immune response. The reason for this is that it can be shown that a considerable increase in the number of antibody containing $B$ cells has occurred in the submucosa by the second day (Blandford, Cureton and Heath, 1971) and there is indirect evidence that these cells are producing specific anti-Sendai antibody by the third day of the infection (Blandford and Heath, 1972). Although it cannot be said that there is a synchronous appearance of the various host responses to the infection, it is apparent that they are all induced at roughly the same time early in the infection.

The relative importance of the various host defences

Although it is probable that both the non-specific defences (interferon and inflammation) and the specific immune response all play some part in terminating these infections, it was of interest to attempt to find out which had the predominant role. Pertinent information was obtained by treating Sendai-infected mice with the immunosuppressant drug cyclophosphamide (Robinson, Cureton and Heath, 1969). The effect of this treatment was

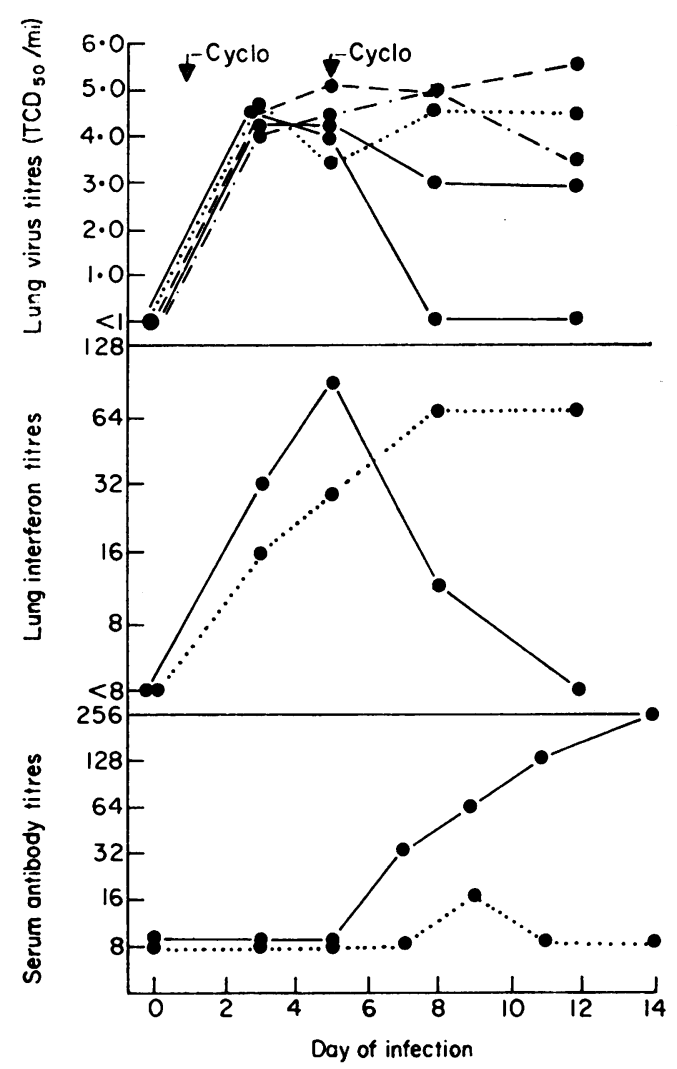

FIG. 3. Effect of cyclophosphamide on virus growth, interferon and serum antibody production in Sendaiinfected mice. - $=$ Untreated. $---=400 \mathrm{mg} / \mathrm{kg}$. $\ldots . .=200 \mathrm{mg} / \mathrm{kg}$. $\quad-.-=100 \mathrm{mg} / \mathrm{kg}$. $-=50 \mathrm{mg} / \mathrm{kg}$. (Reproduced by permission of the Journal of Medical Microbiology). 
dramatic since it converted what is normally a mild inapparent infection into a fatal pneumonic illness. The sequence of events occurring in this altered infection is summarized in Fig. 3. The most obvious difference between the disease process in normal and cyclophosphamide-treated mice is the inability of the immunosuppressed animals to eradicate virus from their lungs. The apparent cause of this is the absence of the immune response, which manifests as nonappearance of serum antibody, and also by the histological finding that mononuclear cells do not appear in underlying areas of affected mucosa. In contrast to this, the non-specific defence mechanisms show no diminution but are exaggerated. Interferon production, for example, continues until the death of the animal and inflammatory changes are not only more intense in the submucosa but have now extended to the alveoli. These exaggerated non-specific defence mechanisms on their own are obviously quite incapable of eradicating the virus.

\section{The humoral immune response}

The appearance of large numbers of immunoglobulin-containing lymphocytes in the submucosa on the second day of the infection (Blandford et al., 1971), the demonstration that these cells are producing specific antibody by the third day (Blandford and Heath, 1972) and the disastrous results that

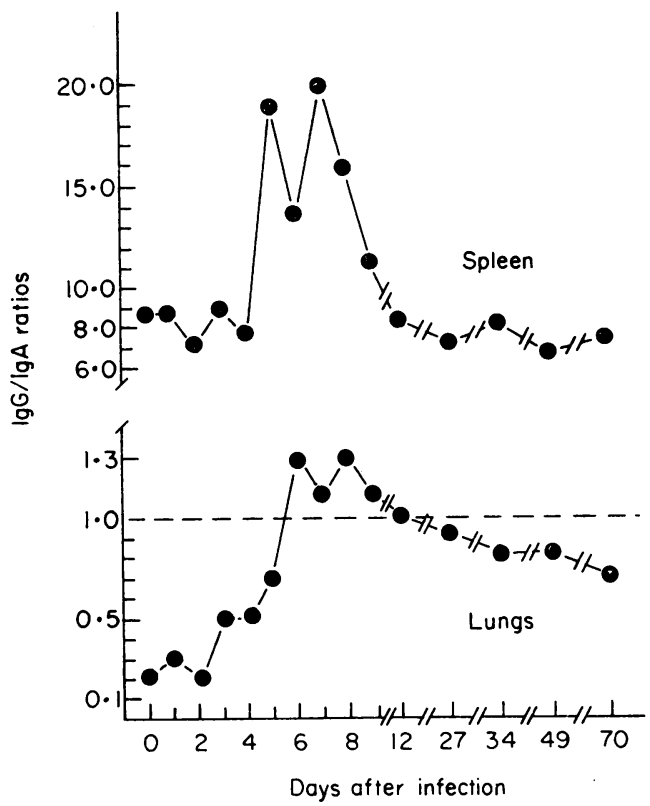

FIG. 4. Ratios of IgG- and IgA-containing cells in the spleen and respiratory submucosa of mice at various intervals after infection with Sendai virus. (Reproduced by permission of Immunology). ensue if this submucosal response is prevented by cyclophosphamide, all suggest that B lymphocytes are of prime importance in terminating the infection.

A study of the various immunoglobulin classes produced by these B cells was of interest (Blandford and Heath, 1974) and the results are summarized in Fig. 4. Before inoculation, the few IgA- and IgGcontaining cells in the submucosa were present in the expected ratio of $5: 1$. However, at the height of the infection, when there was an appreciable increase in the numbers of both kinds of cell, the ratio was approximately $1: 1$. IgA-containing cells did not again appear in excess until the thirtieth day after inoculation. These findings clearly show that the dominant humoral response to this damaging viral infection is the local induction of IgG rather than IgA class antibody.

\section{The cellular immune response}

Not all of the mononuclear cells accumulating in the submucosa could be shown by immunofluorescence to have Ig surface markers and it is not unreasonable to assume that some of these are $T$ cells. Indeed it can be shown by ${ }^{51} \mathrm{Cr}$ release methods that $T$ cells sensitized to Sendai antigens can be detected in the spleen early in the infection, with their activity reaching a peak on the sixth day. It can reasonably be presumed that cells with similar activity appear in the respiratory mucosa. The cytotoxic activity of normal and sensitized spleen cells against Sendai-infected target calls is a complex phenomenon (Anderson et al., 1977) which will not be detailed here. It was, however, intriguing to find out whether these sensitized $T$ cells played any role in the primary defence against the Sendai virus. It is, for example, well established that $\mathrm{T}$ cell-mediated immunity is essential for efficient recovery from infection by poxviruses and herpesviruses but this type of immunity has been shown to be of no importance in recovery from enteroviral infection. The role of cell-mediated immunity (CMI) in recovery from respiratory myxoviral infection is not clearly understood. Suzuki, Ohya and Ishida (1974) have shown that administration of either antilymphocyte serum or antilymphocyte globulin reduces the mortality of mice infected with a pneumonic strains of influenza. The findings suggest that $T$ cells have an immunopathological effect on the lung infection. Virelizier (1975) has shown that thymus-deprived mice show increased susceptibility to infection with PR8 virus and he has attributed this to the absence of the helper effect of $T$ cells on antibody production.

It was clearly of interest to observe the effect of $T$ cell deprivation on the Sendai infection which is restricted to the mucosa. Accordingly, mice were thymectomized, irradiated and reconstituted with 
bone marrow cells (TXBM) and subsequently infected with Sendai virus. The results of this experiment (Anderson and Heath, to be published) were briefly as follows: The thymus-deprived mice were found to be more susceptible to the infection since they developed pneumonitis. The immunosuppressant effect was not nearly as marked as that produced by cyclophosphamide, as none of the mice died and none became particularly ill. It is not clear why the defences were defective in these TXBM mice. The serum antibody response was normal and large numbers of plasma cells appeared in the submucosa. Thus, although the $\mathrm{T}$ cell-mediated response is not essential for recovery, it nevertheless has a role in defence.

\section{The macrophage}

Bacterial pneumonia is a well known complication of viral infection of the upper respiratory tract. Possible reasons for this are disruption of the 'mucociliary escalator' due to damage of the mucosal cells and the inflammatory oedema induced by this damage. Another possibility is that virus infection impedes the antibacterial activity of alveolar macrophages.

It has been shown by immunofluorescence that mouse peritoneal and alveolar macrophages can be infected by Sendai virus but infectious virus is not released from these cells (Mims and Murphy, 1973). It has also been shown that Sendai-infected mice are much less efficient than normal mice in clearing deliberately inoculated Haemophilus influenzae organisms from their lungs (Degré and Glasgow, 1968). This impaired clearance was associated with the development of pneumonia which could not be induced by either virus or bacterium when each was inoculated alone.

Further studies with the Sendai model should be capable of more precisely defining the defect in anti-bacterial defences occurring in upper respiratory tract infection.

\section{Discussion}

Sendai virus infection of mice is a useful model of acute respiratory viral infection of man since the disease process is restricted to the lining of the airway. It is thus more satisfactory than the more commonly used influenza infections which induce pneumonic illnesses. Fundamental concepts of the nature of acute respiratory viral infection can be gained by use of this Sendai model. The data obtained are probably applicable to all respiratory viral infections. Indeed, virtually identical results have been obtained with the Kunz strain of influenza virus, which grows well in the respiratory tract of mice without producing pneumonic changes (Raut et al., 1975).

In terms of pathogenesis, the early stages of the infection are unremarkable. Infected mucosal cells are destroyed in a manner similar to that seen in tissue culture systems infected by cytopathic viruses. What is remarkable is the fact that this infective process is rapidly stopped and the damaged mucosa repaired. The efficient defence processes which bring about the termination of the infection are of considerable importance. This is because an unremitting destructive infection of the respiratory mucosa would so expose the alveoli to both pathogenic and commensal bacteria, that a fatal outcome to the disease would be probable.

The effective defence of the respiratory system against viral infection is affected by a number of factors including the immune response, macrophages, interferon and inflammatory changes. It appears that the immune response is the most important of these, particularly its humoral arm. It is certain that lymphoid cells of the B series, which produce antibodies in the immediate vicinity of affected areas of mucosa, have the predominant role in terminating the infection. It is also possible that the B cells that produce IgG class antibodies are more important that those that produce $\operatorname{IgA}$. In the convalescent period, however, re-infection is prevented by secretory IgA in the bronchial mucus.

Lymphoid cells of the $\mathrm{T}$ series become sensitized to Sendai antigen during the course of the infection These cells do not seem to have an important role in defence against the infection but they are not totally unimportant as they appear to have a 'helper' effect on the humoral response.

The Sendai model can be used to evaluate the role of macrophages in acute respiratory viral infection. Evidence has been obtained which suggests that Sendai virus can impair the antibacterial activity of alveolar macrophages thus increasing the probability of the development of secondary bacterial pneumonia.

The crucial event in these common infections must be the appearance of mononuclear cells of the lymphoid series in the respiratory submucosa. If their rate of accumulation at this site could be enhanced then the severity of the infection would be bound to be reduced. This would be particularly true of symptomatic secondary viral infections. Immunopotentiation might thus be an additional aim of future respiratory viral therapy and prophylaxis.

\section{Acknowledgments}

The work referred to in this paper was undertaken by a number of colleagues including Drs T. W. E. Robinson, R. J. Cureton, G. Blandford, S. Raut, Mrs Hurd and Mrs Anderson. 


\section{References}

Anderson, M.J., Bainbridge, D.R., Pattison, J.R. \& HeAth, R.B. (1977) Cell mediated immunity to influenza infection in mice. Infection and Immunity, 15, 239.

Andrewes, C.H., Laidlaw, P.P. \& Smith, W. (1934) The susceptibility of mice to the viruses of humans and swine influenza. Lancet, ii, 859.

Blandford, G., Cureto., R.J.R. \& Heath, R.B. (1971) Studies of the immune response in Sendai virus infection of mice. Journal of Medical Microbiology, 4, 351.

BLANDFord, G. \& Heath, R.B. (1972) Studies on the immune response and pathogenesis of Sendai virus infection of mice. I. The fate of viral antigens. Immunology, 22, 637.

BlandFord, G. \& HeATH, R.B. (1974) Studies on the immune response and pathogenesis of Sendai virus infection of mice. II. The immunoglobulin class of plasma cells in the bronchial sub-mucosa. Immunology, 26, 667.

Degré, M. \& Glasgow, L.O. (1968) Synergistic effect in viral-bacterial infection. I. Combined infection of the respiratory tract in mice with parainfluenza and hemophilus influenza. Journal of Infectious Diseases, 118, 449.

FRANCIS JR, T. \& STUART-HARRIS, C.H. (1938) Studies on the nasal histology of epidemic influenza virus infection in the ferret. Journal of Experimental Medicine, 68, 789.
Hers, J.F.Ph., Masurel, N. \& Mulder, J. (1958) Bacteriology and histopathology of the respiratory tract and lungs in fatal Asian influenza. Lancet, ii, 1141.

Mims, C.A. \& MuRPhY, F.A. (1973) Parainfluenza virus Sendai infection in macrophages, ependyma, choroid plexus, vascular endothelium and respiratory tract of mice. American Journal of Pathology, 70, 315.

Raut, S., Hurd, J., Cureton, R.J., Blandford, G. \& HeATH, R.B. (1975) The pathogenesis of infections of the mouse caused by virulent and avirulent variants of an influenza virus. Journal of Medical Microbiology, 8, 127.

Robinson, T.W.E., Cureton, R.J.R. \& Heath, R.B. (1968) Pathogenesis of Sendai virus infection in the mouse lung. Journal of Medical Microbiology, 1, 89.

Robinson, T.W.E., Cureton, R.J.R. \& Heath, R.B. (1969) The effect of cyclophosphamide on Sendai virus infection on mice. Journal of Medical Microbiology, 2, 137.

Suzuki, F., OHYA, J. \& IsHIDA, N. (1974) Effect of antilymphocyte serum on influenza virus infection in mice. Proceedings of the Society for Experimental Biology and Medicine, 146, 78.

VIRELIZIER, J.-L. (1975) Host defences against influenza virus: the role of anti-haemagglutinin antibody. The Journal of Immunology, 115, 434. 